\title{
TIPOLOGÍA FONOLÓGICA DEL TIMOTE
}

Anita Arrieta

\begin{abstract}
RESUMEN
Se presentan en este artículo las conclusiones obtenidas, en materia de fonología, a partir del análisis de los datos disponibles sobre el timote, extinta lengua de la Cordillera de los Andes Venezolanos. Para ello, se sistematizan los resultados bajo la forma de rubros fonológicos según la encuesta tipológica elaborada por Constenla (1991:183-185) (con la misma numeración).
\end{abstract}

\begin{abstract}
Once the data available was analyzed, the phonological conclusions arrived at for timote, an extinct language of the Venezuelan Andes, are presented. Results are systematized under phonological items according to the typological survey presented by Constenla (1991:183-185) (keeping the same numerical sequence).
\end{abstract}

\section{Introducción}

Como parte de una contribución al conocimiento de la actualmente extinta lengua timote, que se habló en la Cordillera de los Andes Venezolanos, se presentan en este artículo las conclusiones obtenidas, en materia de fonología, a partir del análisis de los datos disponibles sobre esta lengua. Para ello, se sistematizan los resultados bajo la forma de rubros fonológicos de la encuesta tipológica elaborada por Constenla (1991:183-185) y se utiliza para cada rubro la misma numeración empleada en la encuesta ya mencionada.

\section{Fonología: rasgos tipológicos}

\subsection{Rubros fonológicos de la encuesta}

La referencia a los entornos se hace en función de la interpretación de la fonología del timote que se ha derivado del análisis efectuado (véase el cuadro de fonemas). Los ejemplos de los entornos, en cambio, se dan en la grafía de las fuentes. Por ejemplo, si se da el entorno $t k$ y la forma citada es tigús 'higo', se hace referencia a una aparición de i entre los fonemas / t / y / k /, el segundo de los cuales tiene una manifestación $[\mathrm{g}$ ] representada por la fuente por la letra $g$. 


\subsubsection{Rubro 43. Oposición entre una vocal alta y una media en la serie anterior}

En timote se da oposición entre la vocal alta / i / y la media / e / de la serie anterior.

Al observar los datos, la letra $i$ tiene gran frecuencia y alterna con la letra $e$, de menor frecuencia, por lo que pareciera que estas letras representan un mismo fonema.

Ejemplos:

$$
\begin{aligned}
& \text { 'hermano' } \\
& \text { 'garbanzo' } \\
& \text { 'lunes' } \\
& \text { 'puerta' } \\
& \text { 'cabello' }
\end{aligned}
$$

kushís, kushés

chu-fués, fuix

numis, numes

ustate, stati

meshé mishú, meshi mishu

Sin embargo, los entornos de aparición de esas letras resultaron sumamente parecidos. Ejemplos:

\section{Entornos de e}

$\begin{array}{lll}\mathrm{t} & \mathrm{b} & \text { tebék } \\ \mathrm{t} & \mathrm{k} & \text { guateke } \\ \mathrm{t} & \mathrm{s} & \text { tesasote } \\ \mathrm{t} & \mathrm{m} & \text { tempey } \\ \mathrm{t} & \mathrm{r} & \text { naksterén } \\ \mathrm{k} & \mathrm{S} & \text { shuxashuskes } \\ \mathrm{s} & \# & \text { munse } \\ \mathrm{s} & \mathrm{k} & \text { misék } \\ \mathrm{r} & \# & \text { sore }\end{array}$

\section{Entornos de i}

$\begin{array}{lllll}\text { 'arriba' } & \text { t } & \text { b } & \text { tibís } & \text { 'los' } \\ \text { 'ir' } & \text { t } & \text { k } & \text { tigús } & \text { 'higo' } \\ \text { 'raíces' } & \text { t } & \text { s } & \text { tisép } & \text { 'leña' } \\ \text { 'cargador de muertos' } & \text { t } & \text { m } & \text { ti-m-beuch } & \text { 'madera' } \\ \text { 'frío' } & \text { t } & \text { r } & \text { tirabú } & \text { 'pan negro' } \\ \text { 'queso' } & \text { k } & \text { s } & \text { kis } & \text { 'pulga' } \\ \text { 'caldo' } & \text { s } & \# & \text { titsí } & \text { 'apio' } \\ \text { 'ligero' } & \text { s } & \text { k } & \text { musik } & \text { 'mañana' } \\ \text { 'embudo' } & \text { r } & \# & \text { surí } & \text { 'frijol cultivado' }\end{array}$

Por lo tanto, se determinó la existencia de un fonema / i / y de un fonema / e /. Llama la atención en los entornos el hecho de que ante la vocal $i$ los sonidos nasales tengan una alta frecuencia, mientras que ante la vocal $e$ aparecen únicamente nueve casos.

Ejemplos:

\section{Entornos de e}

$\begin{array}{lll}\mathrm{m} & \mathrm{s} & \text { meshé mishú } \\ \mathrm{n} & \# & \text { sané } \\ \mathrm{n} & \mathrm{k} & \text { tkenék } \\ \mathrm{n} & \mathrm{s} & (5) \text { kunest } \\ \tilde{\mathbf{n}} & \mathrm{m} & \text { kuñém }\end{array}$

\section{Entornos de i}

‘péineme!'
'niño'
'loro'
'pies'
'manos'

$\begin{array}{ll}\mathrm{m} & \# \\ \mathrm{~m} & \mathrm{~b} \\ \mathrm{~m} & \mathrm{t} \\ \mathrm{m} & \mathrm{k} \\ \mathrm{m} & \mathrm{s} \\ \mathrm{m} & \mathrm{n} \\ \mathrm{m} & \mathrm{r} \\ \mathrm{n} & \# \\ \mathrm{n} & \mathrm{b} \\ \mathrm{n} & \mathrm{k} \\ \mathrm{n} & \mathrm{s} \\ \mathrm{n} & \mathrm{r}\end{array}$

$\begin{array}{lll} & \text { kismí } & \text { 'tarde' } \\ \text { mibú } & \text { 'huevos' } \\ \text { (4) mitaifok } & \text { 'bello' } \\ \text { (5) mikuch } & \text { 'tierra' } \\ \text { (6) misék } & \text { 'ligero' } \\ \text { (6) kusminá } & \text { 'sombrero' } \\ \text { (2) mirús } & \text { 'berro' } \\ \text { (4) kiní } & \text { 'legaña' } \\ \text { manibék } & \text { 'miércoles' } \\ \text { (4) nikitao } & \text { 'planta resinosa' } \\ \text { (11) nís } & \text { 'sentarse' } \\ \text { nirú } & \text { 'polvo' }\end{array}$




\subsubsection{Rubro 44. Oposición entre una vocal alta y una media en la serie posterior}

Sí existe oposición entre la vocal alta / u / y la vocal media / o /, en la serie posterior, a pesar de que alternancias como las siguientes parecían indicar lo contrario:

$\begin{array}{ll}\text { 'muro de piedras' } & \text { katafú, katafó } \\ \text { 'viejo' } & \text { kunduk, kundok } \\ \text { 'estómago' } & \text { kitú, kitó } \\ \text { 'sed' } & \text { marakuy, marakoy } \\ \text { 'boca' } & \text { ma-kabú, ma-kabó } \\ \text { 'vecino' } & \text { muskite, moskite } \\ \text { 'puerco' } & \text { purkú, purko (castellanismo) } \\ \text { 'yuca' } & \text { tosmús, kiu-tokmósh }\end{array}$

Las alternancias anteriores, como ya se dijo, parecían ser indicadores de que esas letras representaran un mismo fonema. No obstante, los entornos de esas letras resultaron ser, como en el caso de las letras $i$ y $e$ del rubro 43, muy similares. Sí fue notable el hecho de que la frecuencia de aparición de la letra $u$ fuera muchísimo mayor que el de la letra $o$ (151 palabras con $o$ y 303 con $u$ ).

Ejemplos de similitud de entornos:

\section{Entornos de u}

\begin{tabular}{|c|c|c|c|c|}
\hline $\mathrm{p}$ & \# & koipú & 'sombrero' & $\mathrm{p}$ \\
\hline $\mathrm{t}$ & \# & kuatú & 'coral' & $\mathrm{t}$ \\
\hline $\mathrm{t}$ & $\mathrm{k}$ & tug & 'muro' & $\mathrm{t}$ \\
\hline $\mathrm{k}$ & s & kus & 'mío' & $\mathrm{k}$ \\
\hline $\mathrm{S}$ & \# & nasasú & 'dar' & $\mathrm{s}$ \\
\hline$S$ & $\mathrm{t}$ & sut & 'santo' & $\mathrm{s}$ \\
\hline & $\mathrm{k}$ & sukxos & 'tronco' & $\mathrm{s}$ \\
\hline & \# & kuibimú & 'beber' & $\mathrm{m}$ \\
\hline
\end{tabular}

\section{Entornos de o}

\subsubsection{Rubro 45. Oposición por redondeamiento entre vocales no anteriores (centrales o} posteriores de una misma altura)

En los datos se encuentran con extremada frecuencia alternancias entre las secuencias de letras ue y eu entre sí y con las letras que en la ortografía castellana representan vocales simples.

Ejemplos:

heup, hop

teuk, tek, tok, tuk

kunam, kunan, kuneu-kneu

shimpué, shombeuch, shombuch, shimbú

ti-m-puek, timbéu, ti-m-puče

musipuek, musipuk

$\begin{array}{ll}\text { tapó } & \text { 'tierra' } \\ \text { ubito } & \text { 'árbol' } \\ \text { nuntok } & \text { 'deforme' } \\ \text { mokosh } & \text { 'comprar' } \\ \text { sarsó } & \text { 'mazamorra' } \\ \text { tesasote } & \text { 'raíces' } \\ \text { tchok-tchabón } & \text { 'mediodía' } \\ \text { shamó } & \text { 'gavilán' }\end{array}$

'tener'

'ir'

'mujer'

'agua'

'madera'

'pueblo' 
De gran interés en relación con estas alternancias es el reconocimiento por parte de Jahn, el recolector de la información que parece haber tenido mayores conocimientos sobre lenguas, de sonidos vocálicos inexistentes en castellano y transcritos por él por medio de las letras $\ddot{u}, \ddot{o}$ y que, según su opinión, representarían los mismos sonidos que en la ortografía alemana. La $\ddot{o}$ alterna con otras letras como $o, i, u, e$ y la $\ddot{u}$ alterna con $i u, i, u$.

Ejemplos:

shömbuch

ksöks, kasoks

shnöpa, shnopa, shirup

ti-shöp, t-ssep

narúpchu kfeu, naru-chfeu, naru-chkfeu 'agua' (compárese con la serie precedente de ejemplos)

'cocodrilo'

'fuego'

'leña'

'luna'

Todo lo anterior parece indicar que existía en timote por lo menos una vocal de una cualidad intermedia entre la de las anteriores y central castellanas (siempre no redondeadas) y las posteriores castellanas (siempre redondeadas) o la central castellana (baja) y las no centrales (medias o altas). La impresión de Jahn de que se trataba de sonidos anteriores redondeados no parece ser muy exacta a partir de consideraciones areales. En efecto, en la Sudamérica Atlántica son totalmente comunes las vocales posteriores no redondeadas y las centrales medias o altas, en tanto que las anteriores redondeadas sólo aparecen ocasionalmente como alófonos de otras vocales en entornos muy restringidos. La confusión de unas con otras, por su cualidad intermedia en relación con los fonemas vocálicos castellanos, fue frecuente entre recolectores de información sin preparación fonética. Para citar un caso, Celedón (1886) empleó el símbolo ö, según él, con el mismo valor que en alemán, para representar sonidos de las lenguas, relativamente próximas, de la Sierra Nevada de Santa Marta, que, en transcripciones hechas por lingüistas actuales son más bien centrales medias o altas.

En consecuencia, lo más acertado pareciera ser postular un fomena posterior o central con realizaciones tanto altas como medias $/ \Varangle /$, lo cual explicaría las múltiples maneras en que se representó. De este modo, todos los casos de alternancia señalados se considerarán manifestaciones de este fonema. Además, en vista de lo habitual de su alternancia con letras que representan vocales simples y de que no parecen haber existido en la lengua grupos vocálicos del mismo tipo, todas las apariciones de la secuencia 'eu' se interpretarán como representaciones de dicho fonema.

\subsubsection{Rubro 46. Oposición abierta / cerrada entre vocales de una misma altura y una misma} serie: como / $\mathrm{i} /$ frente $a / i / y / e /$ frente a $/ \varepsilon /$ en la serie anterior

Los datos no ofrecen ningún indicio, por pequeño que sea, de que existieran oposiciones de este tipo. En consecuencia, se considera que la lengua no las presentaba. Esto está de acuerdo con lo que sucede en general en las lenguas geográficamente próximas, sean de las Áreas Culturales Intermedia, Amazónica o Caribe. De las treinta y ocho lenguas del Área Amazónica tomadas en cuenta por Constenla (1991: 205-8), sólo dos, el yaruru y el piaora 
presentan este rasgo. De las cuatro del Área Caribe, ninguna lo presenta. En cuanto al Área Intermedia, de las diecisiete lenguas de Colombia y Venezuela tomadas en cuenta por el mismo autor, sólo una, situada a gran distancia (costa pacífica noroeste de Colombia), el huaunana, lo presenta.

\subsubsection{Rubro 47. Oposiciones entre vocales por nasalidad}

En los datos, numerosas palabras presentan alternancias entre consonantes nasales finales de sílaba (normalmente homorgánicas de la consonante siguiente) y cero.

Ejemplos:

$\begin{array}{ll}\text { shimpué, shipué } & \text { 'agua' } \\ \text { funt, xut } & \text { 'alacrán' } \\ \text { keún, kuu } & \text { 'dormir' } \\ \text { estuce, ismtuch } & \text { 'llama' } \\ \text { xok, köngk } & \text { 'nariz' } \\ \text { tump, tuup } & \text { 'piedra' } \\ \text { sharin, chivatsari } & \text { 'cerca' }\end{array}$

Por otra parte, se dan también bastantes casos de alternancia entre la vibrante simple $r$ y la nasal $n$ en posición inicial de sílaba, como se observa en:

$\begin{array}{ll}\text { gua-sharé } & \text { 'hijo' } \\ \text { sané } & \text { 'niño' } \\ \text { shirup, shnopa } & \text { 'fuego' }\end{array}$

Estos tipos de alternancias sugieren la presencia de nasalidad vocálica distintiva, que habría sido interpretada en unos casos como consonante nasal final de sílaba y habría dejado de percibirse en otros. Podría haber sucedido que en timote, como en algunas lenguas chibchas y chocoes (Abarca González 1985, Harms 1984), como parte de la realización de la nasalidad vocálica se diera facultativamente un segmento fonético consonántico nasal. Los casos de alternancia entre $r$ y $n$ podrían reflejar la existencia de una regla de expansión de la nasalidad vocálica a segmentos consonánticos sonoros contiguos como se da también en lenguas de las familias antes mencionadas (Harms 1984; Constenla 1984). De acuerdo con lo anterior, se considera que el timote presentaba nasalidad vocálica distintiva.

No obstante, cabría una interpretación alternativa de la variación entre finales y cero: la oposición entre los fonemas nasales / $\mathrm{n} / \mathrm{y} / \mathrm{m} / \mathrm{se}$ habría neutralizado al final de sílaba y el archifonema resultante se habría realizado por medio de una consonante nasal homorgánica de la consonante siguiente, por medio de $[\mathrm{m}]$ o [ $\mathrm{n}$ ] ante pausa $\mathrm{o}$, facultativamente, como nasalización de la vocal precedente. Esta explicación no se ha adoptado porque no ofrece la ventaja de la primera de dar cuenta también de la alternancia entre $r$ y $n$. 


\subsubsection{Rubro 48. Oposiciones entre vocales por cantidad}

Los casos en los que pareciera haber vocales largas son los siguientes:

$\begin{array}{ll}\text { 'quejarse' } & \text { aá } \\ \text { 'persona' } & \text { kaak } \\ \text { 'tabaco casi líquido' } & \text { moo } \\ \text { 'adormecido' } & \text { muu } \\ \text { 'dulce' } & \text { tiboó } \\ \text { 'lentamente' } & \text { timafaá }\end{array}$

Sin embargo, las alternancias con vocales breves no están bien establecidas. Por consiguiente, se considera que la lengua no presentaba vocales largas.

Aparecen otros casos, con alargamiento de la vocal $\mathrm{u}$, en los que alternan vocales largas y consonantes nasales.

Ejemplos:

$\begin{array}{ll}\text { 'piedra' } & \text { tuup, tump } \\ \text { 'olla' } & \text { nayuu, nayún, nayuú } \\ \text { 'frijoles' } & \text { shituuk, sihtuuk, sigtún }\end{array}$

Esta alternancia sugiere la existencia de vocales largas, pero también podría tratarse de una confusión, debido a las transcripciones de los datos, entre las grafías de la $m$ y de la $u$. También estas alternancias pueden interpretarse, de acuerdo con lo propuesto en el rubro 47, como casos de vocales nasales que en algunos casos habrían sido interpretados como consonante nasal.

Dado que los indicios son muy tenues, lo más prudente pareciera ser no postular la existencia de oposiciones cuantitativas entre las vocales, a pesar de que el rasgo en cuestión se dé en guajiro y casi seguramente en jirajara, lengua en la que las vocales dobles se presentan con mucha frecuencia.

\subsubsection{Rubro 49. Oposiciones tonales}

Los datos, tal como han sido transcritos, no permiten determinar oposiciones tonales. En el Área Intermedia presentan oposiciones tonales el paya, el boruca, el bribri, el cabécar, el moverse, el bocotá y el tunebo. En el Área Amazónica se dan en la familia tucana mientras que en las Áreas Peruana y Caribe tales oposiciones no se presentan.

\subsubsection{Rubro 50. Presencia de oclusivas glotalizadas no labiales}

No hay indicios de que en timote se presentaran oclusivas glotalizadas. Este rasgo, además, no se da en ninguna lengua vecina y, más bien, opone las lenguas de las Áreas Intermedia, Caribe y Amazónica (entre las que muy pocos presentan tales fonemas) a las del Área Peruana (como ciertos dialectos del quechua, el aimara y el uru-chipaya) donde estos fonemas constituyen una serie importante. 


\subsubsection{Rubro 51. Presencia de una oclusiva glotalizada labial}

$\mathrm{Al}$ parecer, no se daban en timote (véase el rubro anterior).

\subsubsection{Rubro 52. Presencia de por lo menos una implosiva}

No hay indicios en los datos de la presencia de consonantes implosivas. Este rasgo no se presenta en la muestra de lenguas de las Áreas Intermedia, Caribe y Amazónica analizada por Constenla para la elaboración de la encuesta.

\subsubsection{Rubro 53. Presencia de oposiciones por aspiración entre oclusivas}

No existe ningún indicio en los datos del timote de este tipo de oposición, que de todos modos era poco frecuente en las Áreas Intermedia, Caribe y Amazónica.

De hecho, ninguna de las lenguas habladas en el Occidente de Venezuela parece haberla presentado.

\subsubsection{Rubro 54. Condición fonemática de la oclusión glotal}

No hay indicios en los datos de presencia del fonema / /, por lo que lo más probable es que no se diera. En este caso, los conocimientos existentes sobre la tipología areal no permiten ser muy concluyentes al respecto, pues en las vecindades del timote se dan tanto lenguas que lo presentan como lenguas que carecen de él.

\subsubsection{Rubro 55. Inclusión de un fonema / $p /$ (oclusivo simple, bilabial, sordo)}

En los datos, abundan las alternancias entre las letras $p, b, v, w$, y la secuencia de letras $g u$, que representa articulaciones labiales no nasales.

En los siguientes ejemplos, $p$ y $b$ alternan cuando están precedidas por la letra $m$ (es decir, por vocales nasales).
shimbú, shimpú
'agua'
ti-m-beuch, ti-m-puek
'madera'
timbós, timpósh
'tubérculo'

Se presentan, por otra parte, alternancias en posición intervocálica o en posición inicial absoluta entre $b, v, g u$ y $w$. Ejemplos:

$\begin{array}{ll}\text { es-kivit, kibit } & \text { 'bastón' } \\ \text { chikibú, chikiguó, chikive } & \text { 'azúcar' } \\ \text { sbúts, guéuch } & \text { 'calor' } \\ \text { chi-kivu, chi-kibuk } & \text { 'fango' } \\ \text { wó, guó, bo } & \text { 'ya' }\end{array}$


En vista de estas alternancias, resulta evidente que las letras $v, w, b$ y $g u$ no representaban fonemas distintos. En el caso de $p$, a pesar de que alterna con $b$ cuando están precedidas por la letra $m$, la situación es diferente. Aparece más bien opuesta claramente a $b$ (o a los elementos con los que alterna), en todas las otras posiciones.

Ejemplos:

$\begin{array}{ll}\text { pó } & \text { 'cucaracha' } \\ \text { bo, guó, wó } & \text { 'ya' } \\ \text { tsipá } & \text { 'hormigas' } \\ \text { ciba } & \text { 'red para cargar' } \\ \text { civat } & \text { 'yerba santa' } \\ \text { ti-abá } & \text { 'guayaba' } \\ \text { sikapá } & \text { 'huevos' } \\ \text { tabís } & \text { 'diez' } \\ \text { papič } & \text { 'puerta' } \\ \text { čipio } & \text { 'árbol de la familia del caucho' } \\ \text { es-kivit } & \text { 'bastón' } \\ \text { kibí } & \text { 'bastón' }\end{array}$

De las observaciones y ejemplos precedentes se postula la existencia de dos fonemas labiales: uno oclusivo / $\mathrm{p}$ / (que presenta la variante sonora oclusiva [ $\mathrm{b}$ ] cuando se encuentra precedida por vocal nasal y una oclusiva sorda [ $\mathrm{p}$ ] en los demás casos), y uno aproximante / w / que se realizaba por medio de una variante labiovelar semiconsonántica [ $w$ ] o una bilabial [ $\beta$ ]. Estos alófonos parecen haber alternado libremente, aunque [w ] se da con frecuencia ante las vocales no anteriores y [ $\beta$ ] ante las anteriores, como se puede notar en los ejemplos antes señalados.

\subsubsection{Rubro 56. Presencia de uno o más fonemas oclusivos uvulares}

No hay indicios en los datos de la presencia de este tipo de fonema. Esta ausencia se da también en las lenguas de las Áreas Intermedia, Caribe y Amazónica, en oposición al Área Peruana (presentes en quechua y aimara), y a las lenguas mayas de tierras altas en las que sí aparecen fonemas oclusivos uvulares.

\subsubsection{Rubro 57. Presencia de por lo menos un fonema obstruyente (oclusivo o fricativo) labial sonoro o lene}

Los datos indican que sí se daba este fonema (véase el rubro número 55).

1.1.16. Rubro 58. Presencia de por lo menos un fonema obstruyente (oclusivo o fricativo) dental o alveolar sonoro o lene

La letra $d$ tiene muy baja frecuencia en los datos y, con dos excepciones, se presenta únicamente precedida por la letra $n$ (es decir, por vocal nasal, véase el rubro 47). 
También $t$, muy abundantemente, se presenta precedida por $n$. Sin embargo, no hay casos obvios ni de oposición ni de alternancia en los rubros léxicos particulares. En cuanto a la alternancia, se presenta en el formativo de temas nominales que presenta en unos casos la forma $-d o k$ y en otros $-t o k$.

Ejemplos:

kashun-dok

kundók

nuntok

$$
\begin{aligned}
& \text { 'mujer' } \\
& \text { 'viejo' } \\
& \text { 'deforme' }
\end{aligned}
$$

También parece haber habido alternancia en el caso de la palabra escrita teunden o tundéu 'maduro' que podría ser una reduplicación.

Las dos apariciones de la letra $d$ no precedida por $n$ se dan en las palabras suridipa 'pan de maíz' y dú udú 'con, juntamente'.

En vista de lo señalado anteriormente y del paralelismo que se da con la relación entre $p$ y $b$, la conclusión que aquí se plantea es que $t$ y $d$ son manifestaciones de un mismo fonema / $\mathrm{t}$ /. En consecuencia, no se habría dado un fonema obstruyente dental o alveolar sonoro.

\subsubsection{Rubro 59. Presencia de por lo menos un fonema obstruyente (oclusivo o fricativo) ve- lar sonoro o lene}

Existe alternancia entre las letras $k, g, x, h$ y $j$.

En cuanto a $k$ y $g$, parecen funcionar en los datos como $p / b$ y $t / d$, porque gran número de apariciones de $g$ se dan cuando precede una $n$. En vista de esto, se plantean como miembros de un fonema / $\mathrm{k} /$ velar oclusivo sordo.

Ejemplo de alternancias entre $k$ y $g$ : ti-n-guís, ti-n-kuís 'papas'.

Las letras $x$ (empleada por Rivet), $j$ y $h$ (empleadas por los otros autores) también alternan frecuentemente con $k$ y $g$.

Ejemplos en el léxico patrimonial:

$\begin{array}{ll}\text { xok, köng, kong } & \text { 'nariz' } \\ \text { numbuk, numbuh, numbúh } & \text { 'sal' } \\ \text { chingkang, chingkanx } & \text { 'harina' } \\ \text { sukhós, suhxos } & \text { 'tronco' }\end{array}$

Ejemplos en castellanismos:

$\begin{array}{ll}\text { xaguay, kaguay } & \text { 'caballo' } \\ \text { xamis, kamis } & \text { 'camisa' } \\ \text { xarsó } & \text { 'calzón' }\end{array}$

De acuerdo con estos hechos, se daba un único fonema velar $/ \mathrm{k} /$ con cuatro realizaciones: [ $\mathrm{k}$ ] velar oclusiva sorda, $[\mathrm{g}$ ] velar oclusiva sonora, $[\mathrm{x}$ ] velar fricativa sorda y $[\mathrm{h}$ ] laríngea fricativa sorda. 


\subsubsection{Rubro 60. Presencia de por lo menos un fonema fricativo siseante o chicheante sonoro olene}

A partir de los datos es posible determinar la existencia de un único fonema fricativo sordo: / $/$ /.

Lo anterior se deduce de las alternancias frecuentes entre las letras $s, z, t z, t s, c$ y $s s$ que presentan los datos.

Ejemplos:

$\begin{array}{ll}\text { 'cabeza' } & \text { kizán, kishám, hushám, kichan, husham } \\ \text { 'candela' } & \text { tzirup, sirup, chirup } \\ \text { 'látigo' } & \text { ti-marsot, ti-marzot } \\ \text { 'moscas' } & \text { ti-tisán, ti-ti-san, ti-ti-sán, ti-ti-zan. } \\ \text { 'pimiento' } & \text { shikás, chikás } \\ \text { 'tubérculo' } & \text { timbó, timbós, timpósh, timposh } \\ \text { 'dios' } & \text { chés, shes }\end{array}$

Como se puede observar, la frecuencia de las alternancias indica la variación libre entre los sonidos representados por las letras mencionadas y permite postular un fonema / s / cuyas realizaciones comparten el componente fricativo. El modo de articulación puede variar de puramente fricativo a africado y el punto de alveolar a palatoalveolar. La representación ocasional por medio de $s s$ e incluso de $r s$ podría indicar alguna particularidad de la articulación de este fonema. Una hipótesis es que se tratara de una retofleja, lo cual explicaría que fuera percibida por igual como alveolar y palatoalveolar, e incluso se creyera percibir un sonido de tipo $r$ precedente. En relación con esto hay que tomar en cuenta que en los datos es muy poco frecuente la aparición de $r$ ante otra consonante o al final de palabra. La mayor parte de los ejemplos que se encuentran son castellanismos como shaguardén 'aguardiente' o tri-aber 'arveja' o apariciones ante $s, t s, t z$ y $z$ que, de acuerdo con la interpretación aquí propuesta, serían simplemente parte de las manifestaciones del fonema / s /. Existen únicamente tres casos de aparición de $r$ (a final de sílaba interna) ante consonante todos ellos, y uno de aparición a final de palabra de los que no se ha logrado dar cuenta por medio de la explicación propuesta.

\subsubsection{Rubro 61. Oposición por sonoridad o lenidad entre africadas}

De acuerdo con el rubro anterior, las aparentes africadas fonéticas que se dan en los datos (secuencias de letras $c h, t s, t z$ ), son variantes del fonema fricativo / s / De todos modos, se trataba, al parecer, de sonidos sordos. En consecuencia, este rasgo no se presenta.

El tipo de rasgo en cuestión no es frecuente en las lenguas vecinas al timote, no obstante se presenta en por lo menos dos lenguas de la Sierra Nevada de Santa Marta (Colombia): el bíntucua y el guamaca.

\subsubsection{Rubro 62. Oposición por glotalización entre africadas}

Como se señaló en el rubro anterior, la lengua en estudio no presenta sonidos africados y, por lo tanto, tampoco podría presentar oposición por glotalización entre ellos. 
Por otra parte, la oposición por glotalización, como se señaló en 1.1.8, es muy infrecuente en las Áreas Caribe, Amazónica e Intermedia.

\subsubsection{Rubro 63. Oposición por aspiración entre africadas}

En vista de que, al parecer, no existían fonemas africados, esta oposición no podía darse. Este rasgo es muy infrecuente en el Área Intermedia y está ausente en la muestra de lenguas de las Áreas Caribe y Amazónica analizada por Constenla.

\subsubsection{Rubro 64. Presencia de por lo menos un fonema africado alveolar}

De acuerdo con lo planteado en el rubro 61 , los posible sonidos africados como los representados por las grafías $t s$ y $t z$ eran manifestaciones del fonema fricativo / $\mathrm{s} /$.

\subsubsection{Rubro 65. Presencia de por lo menos un fonema africado prepalatal}

De acuerdo con lo planteado en 1.1.18., los posibles sonidos africados como el representado por la grafía ch eran manifestaciones del fonema fricativo / s /.

\subsubsection{Rubro 66. Presencia de un fonema africado lateral}

No hay ningún indicio de la presencia de un elemento de este tipo. No se dan en los datos secuencias de letras como $t l$ o $d l$. De hecho, la letra $l$ es de frecuencia muy baja (véase el rubro 81 ).

\subsubsection{Rubro 67. Presencia de por lo menos un fonema africado retroflejo}

De acuerdo con 1.1.18., los posibles sonidos africados eran manifestaciones del fonema fricativo / s /. Este rasgo no se da en la muestra de lenguas del Área Caribe ni en la del Área Amazónica de Constenla. Por lo que respecta al Área Intermedia, era un fenómeno limitado a lenguas del sudoeste de Colombia y regiones vecinas del Ecuador.

\subsubsection{Rubro 68. Presencia de un fonema $(/ \phi / o / f /)$ fricativo labial sordo}

La letra $f$ aparece muy poco en los datos y alterna con las letras $x, p$, ch y $t$. Ejemplos:

$\begin{array}{ll}\text { 'alacrán' } & \text { fungt, funt, xut } \\ \text { 'anciano' } & \text { ka-nisep, ka-nisef } \\ \text { 'carne' } & \text { chorok, forok } \\ \text { 'cuchara' } & \text { fatak, tafák }\end{array}$

De acuerdo con el análisis que se ha hecho de los sonidos $p, t, k$ y $s$, y debido a las pocas palabras que presentan la letra $f$, no es posible pensar que las variaciones que ilustran los ejemplos anteriores indiquen la posibilidad de que $f$ sea una variable de los fonemas / p /, / t /, /k / o / s /. No hay tampoco suficientes datos como para demostrar que sea un fonema, ya que no se presentan casos en los que este sonido produzca diferencias de significado. 
a) Alternancia con $p$

Podría tratarse de que el fonema / $\mathrm{p} /$ tuviera una realización fricativa como sucede en el caso del fonema / k / en la misma posición (véase el rubro 59). Sin embargo, es difícil proponer esto con certeza debido a las pocas apariciones de alternancias entre las letras $p$ y $f$.

b) Alternancia con $x$

Al haber únicamente un caso, se explica como la posibilidad de que existiera un sonido labializado ante la vocal $u$.

c) Alternancia con ch y con $t$

Por ser casos únicos y por la gran diferencia que existe entre estos sonidos, se explican las alternancias como erratas.

\subsubsection{Rubro 69. Presencia de un fonema (/ / / ) fricativo prepalatal sordo}

Se da la grafía sh y, aparentemente, por lo menos en algunos autores, $c h$ representaba el mismo tipo de sonido fricativo; pero, de acuerdo con lo expuesto en el rubro 60, éste era una manifestación del fonema / s /.

\subsubsection{Rubro 70. Presencia de un fonema (/s/) fricativo retroflejo sordo}

Como se indicó en el rubro 60, hay un solo fonema fricativo / s /. Podría haber tenido realizaciones retroflejas; en consecuencia, no existía una entidad fonológica fricativa retrofleja independiente. Desde el punto de vista areal, en los datos analizados por Constenla, este rasgo en el Área Intermedia aparece sólo en lenguas del sudoeste de Colombia y no se da en las de las Áreas Caribe y Amazónica.

\subsubsection{Rubro 71. Presencia de un fonema (/4/) fricativo lateral sordo.}

No hay indicios de la existencia de un fonema de este tipo. En la muestra de Constenla, sólo una lengua del Área Intermedia (el guatuso de Costa Rica) lo presenta y no se da en las lenguas que tomó en cuenta de las Áreas Caribe y Amazónica.

\subsubsection{Rubro 72. Presencia de fonemas consonánticos nasales bilabial $/ m / y$ alveolar $/ n /$}

En timote se da la oposición entre / $\mathrm{m} / \mathrm{y} / \mathrm{n} /$ restringida a la posición inicial:

Ejemplos:

$\begin{array}{ll}\text { 'allá arriba' } & \text { machú-kupís } \\ \text { 'hombre' } & \text { nachú } \\ \text { 'maguey' } & \text { mabúsh } \\ \text { 'ceniza' } & \text { nabush }\end{array}$

En la posición final, como se señaló en el rubro 47, las apariciones de m y $\mathrm{n}$ al final de sílaba, debido a la alternancia que presentan, se han interpretado como parte de las realizaciones de vocales nasales. 


\subsubsection{Rubro 73. Presencia de un fonema nasal mediopalatal $/ \mathrm{n} /$}

En los datos aparecen apenas ocho casos de la letra $\tilde{n}$. Esto indica que no se daba en timote este rasgo. En el Área Caribe no se presenta tampoco y en el Área Amazónica muy pocas lenguas, como el yaruro y el piaroa, lo tienen. En cuanto al Área Intermedia, está presente en las lenguas del sudoeste de Colombia y de Ecuador (guambiano, cuaiquer, quechua colombiano y quechua ecuatoriano).

\subsubsection{Rubro 74. Presencia de un fonema nasal velar/ g/}

No existe en timote un fonema nasal velar / g /. En posición inicial de sílaba, las únicas letras que se encuentran son la $m$ y la $n$. En cuanto a la posición final, puede haberse dado un sonido $\mathfrak{y}$ (representado por la secuencia $n g$ ) al final de sílaba que, como la $m$ y la $n$ finales de sílaba, habría sido parte de las realizaciones de las vocales nasales, porque la secuencia de letras mencionada se da ante consonante y en variación con $n$.

1.1.33. Rubro 75. Presencia de fonos nasales sordos como realización de fonemas nasales ante / h / o de secuencias de fonemas nasales con / h /

No hay ningún indicio en los datos de la existencia de fonos nasales sordos. En el Área Intermedia éste es un rasgo limitado al Páez del sudoeste de Colombia y a las lenguas misumalpas de Honduras y Nicaragua. El rasgo de todos modos, no se da en las lenguas vecinas al timote.

\subsubsection{Rubro 76. Presencia de grupos iniciales de palabra integrados por una nasal más otra consonante}

No aparecen en la lengua palabras en las que el grupo inicial esté formado por una nasal más otra consonante.

\subsubsection{Rubro 77. Presencia de un fonema aproximante lateral sonoro / / /}

Debido a la poca frecuencia que tiene la letra $l$ en los datos, se ha decidido que esta letra representa una variante del fonema / $\mathrm{r} /$. (Véase el rubro 81).

\subsubsection{Rubro 78. Presencia de un fonema aproximante mediopalatal sonoro /K/}

No se da en timote la presencia de un fonema aproximante mediopalatal sonoro (véase el rubro 81 ).

La letra $l l$ no se presenta en los datos.

\subsubsection{Rubro 79. Presencia de un fonema vibrante simple central/ $\mathrm{I} /$}

En la lengua parece que existió un único fonema vibrante: / I / representado, en sus frecuentes apariciones, por la letra $r$. Ejemplos: 


$\begin{array}{ll}\text { runch } & \text { 'diente' } \\ \text { sore } & \text { 'embudo' } \\ \text { surí } & \text { 'frijol cultivado' } \\ \text { suridipa } & \text { 'pan de maíz' }\end{array}$

En los castellanismos, la $r r$ aparece representada como $r$ o $r r$ (murrú, murú 'burro') y la $l$ como $r$ (murá 'mula'). Esta situación, sumada a la baja frecuencia de $r r$ y $l$ en los datos hacen pensar que los sonidos representados por estas letras fueron variantes del fonema / $\{/$.

Las apariciones de $l$ y $r r$ antes mencionadas son las siguientes:

$\begin{array}{ll}\text { lampako } & \text { 'árbol para la construcción' } \\ \text { klef } & \text { 'pico de ave' } \\ \text { haile } & \text { 'sopa de guisantes' } \\ \text { warre } & \text { 'relámpago' }\end{array}$

La última palabra, la única del léxico en que aparece $r r$, resulta muy problemática porque aparecen también formas como wasre y wawe. Por eso, resulta prácticamente imposible determinar cuál era la consonante que ocupaba la posición intervocálica en ella.

\subsubsection{Rubro 80. Presencia de un fonema vibrante simple lateral/.J/}

Como se indicó en el rubro anterior, hay un sólo fonema vibrante en la lengua. (Véase el rubro 79 ).

\subsubsection{Rubro 81. Presencia de un fonema vibrante múltiple/ $r /$}

Únicamente se dio en la lengua un fonema vibrante / r /. (Véase el rubro 79).

\subsection{Conclusiones acerca de la tipología fonológica del timote}

A partir del análisis expuesto detalladamente bajo la forma de rubros de una encuesta tipológica, se concluye que:

\subsubsection{El sistema fonológico del timote}

Habría incluido las siguientes entidades:

\section{Cuadro 1 \\ Consonantes}

Oclusivas

Fricativas

Nasales

Vibrantes

Semiconson

\section{Bilabial}

$p$

$\mathrm{m}$

W
Alveolar

$\mathrm{t}$

$\mathrm{S}$

n

r
Velar

k 


\section{Cuadro 2}

Vocales

Anteriores

Altas

Medias

Bajas
Centrales

$\mathrm{i}$

$\mathrm{e}$
Posteriores

$\dot{+}$

$\mathrm{u}$

o

Cuadro 3

Fonema covocálico de nasalidad $1 \sim 1$

En el caso de las vocales, es necesario decir que los datos permiten suponer la existencia de vocales nasales y que el sistema vocálico es muy característico del área, ya que lo presentan varias lenguas de las Áreas Intermedia, Caribe y Amazónica.

\subsubsection{Rasgos tipológicos positivos para el timote en materia de fonología}

Los rasgos tipológicos en materia de fonología que resultan positivos para el timote son los siguientes:

a) Oposición entre una vocal alta y una media en la serie anterior

b) Oposición entre una vocal alta y una media en la serie posterior

c) Oposición entre vocales por nasalidad

d) Inclusión de un fonema / p / (oclusivo, bilabial, sordo)

e) Presencia de fonemas consonánticos nasales bilabial / m / y alveolar / n /

f) Presencia de un fonema vibrante simple central / $[/$

En fonología los resultados obtenidos distinguen claramente al timote de las lenguas de las otras áreas y lo vinculan con lenguas como el guajiro y, por consiguiente, con características del Área Caribe tales como: ausencia de oposiciones tonales, ausencia de oposiciones por glotalización, ausencia de aspiración entre oclusivas, ausencia del fonema / / / y ausencia de oposiciones por sonoridad entre las obstruyentes.

\section{Bibliografía}

Celedón, Rafael. 1886. Gramática de la lengua koggaba, con vocabulario y catecismos. París: Léclerc, Editeurs.

Constenla Umaña, Adolfo. 1991. Las lenguas del Área Intermedia: introducción a su estudio areal. San José: Editorial de la Universidad de Costa Rica.

Gleason H.A. 1955. Introducción a la lingüística descriptiva. España: Editorial Gredos.

Jahn, Alfred. 1973. Los aborígenes del Occidente de Venezuela. Caracas: Monte Ávila Editores. 
Mosonyi, Jorge. 1986. "Lenguas indígenas de Venezuela", Boletín de Lingüística de la Escuela de Antropología. Universidad Central de Venezuela. VI, VII: 19-40.

Ritchie Key, Mary. 1968. The grouping of South American Indian Languages. Alemania: Editorial Günter Narr Verlag.

Rivet, Paul. 1927. "La famille linguistique timote". International Journal of American Linguistics. IV: 2-4. 